\title{
Infektionen sind in warmen Monaten häufiger
}

Nach durchschnittlich 1,6\% der operativen Eingriffe kommt es zu Infektionen der Wunde. In den letzten Jahrzehnten ist es zwar gelungen, dieses Risiko durch Hygienemaßnahmen und vorbeugende Medikamentengabe deutlich zu senken. Mediziner der Berliner Charité haben nun jedoch einen Faktor identifiziert, den Ärzte und Klinikpersonal nicht beeinflussen können: das Wetter. Wie sie in einer Studie mit Daten aus 17 Jahren zeigen konnten, treten Wundinfektionen in wärmeren Monaten häufiger auf als in kühleren.

In den Jahren 2000 bis 2016 wurden über das deutsche Krankenhaus-Infektions-Surveillance-Systems (KISS) zwei Millionen Operationen dokumentiert. In deren Folge ist es zu mehr als 32.000 postoperativen Wundinfektionen gekommen. An KISS sind Krankenhäuser aus ganz Deutschland angeschlossen - verwaltet wird das System über die Charité, wo Dr. Seven Johannes Sam Aghdassi und seine Kollegen die Daten auswerteten [1].

Die Angaben zur Wundheilung aus dem KISS verknüpften die Mediziner mit meteorologischen Messdaten des Deutschen Wetterdienstes, wie etwa der Außentemperatur, dem Niederschlag und der Luftfeuchtigkeit. Da diese Parameter stark miteinander korrelierten, konzentrierten sich die Studienautoren bei der Analyse letztlich auf die monatliche Durchschnitts-

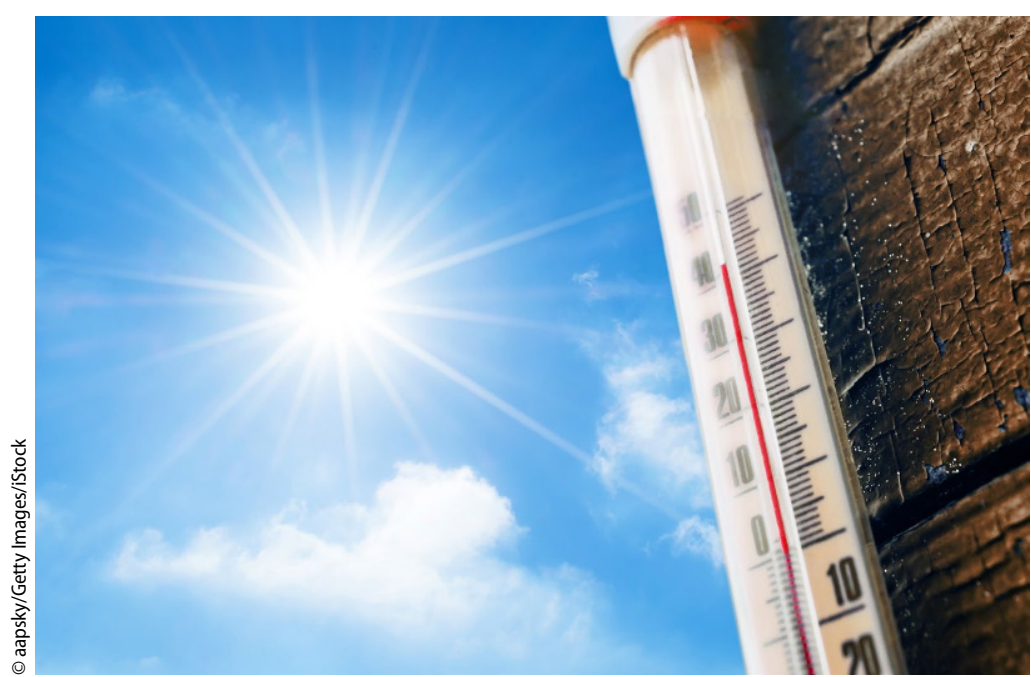

Abb. 1 A Mit jedem Grad Außentemperatur mehr stieg das Risiko für eine postoperative Wundinfektion um $1 \%$ temperatur. Wie sich zeigte, stand diese in einem deutlichen Zusammenhang mit der Zahl der dokumentierten Wundinfektionen: „Grob gesagt nahm mit jedem Grad, um das die Außentemperatur anstieg, das Risiko für eine postoperative Wundinfektion um ein Prozent zu“, erklärt Aghdassi. Bei der Analyse definierter Temperaturbereiche ergab sich zwischen der kältesten Kategorie (weniger als 5 Grad Außentemperatur) und der wärmsten (20 Grad oder mehr) ein Risikozuwachs von $13 \%$ (• Abb. 1).

\section{Gramnegative Erreger stärker temperaturabhängig}

Dabei schienen manche Bakterientypen stärker auf die Außentemperatur zu reagieren als andere. Während sich die Zahl der Infektionen mit grampositiven Erregern nur wenig änderte, nahmen Infektionen mit gramnega- tiven Keimen - wie etwa E.coli-Bakterien - mit steigenden Temperaturen deutlich zu. Auch waren oberflächliche Wundinfektionen deutlich stärker temperaturabhängig als Infektionen in tieferliegenden Wundbereichen.

Die Analyse, an der auch Wissenschaftler des Potsdam Instituts für Klimafolgenforschung beteiligt waren, hat Aghdassi zufolge einen rein explorativen Charakter. „Es handelt sich lediglich um einen ersten Schritt in die Thematik hinein", betont der Berliner Studienleiter. Entsprechend möchte er die Schlussfolgerungen aus seiner Analyse zunächst nur als Hypothese verstanden wissen. Eine Hypothese allerdings, die es angesichts der im Zuge des Klimawandels zu erwartenden Temperatursteigerung weiter zu untersuchen lohnt.

\section{Literatur \\ 1. Aghdassi S J S et al (2019) The Association of Climatic Factors with Rates of Surgical Site Infections: 17 years' data from hospital infection surveillance. Dtsch Arztebl Int 116: 529-536. https://doi.org/10.3238/arz- tebl.2019.0529}

Hinweis des Verlags. Der Verlag bleibt in Hinblick auf geografische Zuordnungen und Gebietsbezeichnungen in veröffentlichten Karten und Institutsadressen neutral.
Quelle: Presseaussendung der Deutschen Gesellschaft für Innere Medizin e.V. (DGIM) 